\title{
Thermal Analysis of SC Quadrupoles in Accelerator Interaction Regions
}

\author{
Igor Novitski, Alexander V. Zlobin
}

\begin{abstract}
This paper presents results of a thermal analysis and operation margin calculation performed for $\mathrm{NbTi}$ and $\mathrm{Nb}_{3} \mathrm{Sn}$ low-beta quadrupoles in collider interaction regions. Results of the thermal analysis for NbTi quadrupoles are compared with the relevant experimental data. An approach to quench limit measurements for $\mathrm{Nb}_{3} \mathrm{Sn}$ quadrupoles is discussed.
\end{abstract}

Index Terms - Superconducting quadrupole, interaction region, operation margin, thermal analysis, temperature margin, quench limit.

\section{INTRODUCTION}

$\mathrm{T}$ The final beam focus in collider interaction regions (IR) is provided by superconducting low-beta quadrupoles placed next to the detector on both sides of the interaction point. The first generation of IR quadrupoles (IRQ) based on $\mathrm{NbTi}$ superconductor are being used in Tevatron [1] and in LHC [2], [3]. A design study of the second generation IR quadrupoles for the LHC luminosity upgrade has been started recently in the framework of U.S. LHC Accelerator Research Program (LARP) [4]. These magnets will use $\mathrm{Nb}_{3} \mathrm{Sn}$ superconductor and provide larger aperture and larger operation margin to increase the LHC luminosity. Superconducting low-beta quadrupoles based on $\mathrm{NbTi}$ or $\mathrm{Nb}_{3} \mathrm{Sn}$ coils are also considered for the ILC IR [5].

Secondary particles coming from interaction point deposit energy in IR quadrupoles and, in particular, in their coils. This energy deposition will cause a coil temperature rise and may lead to a premature magnet quench if coil cooling conditions are not sufficient. To prevent quenches the coil temperature has to be kept below the superconductor critical temperature at given transport current. Moreover, additional operation margin is usually required for the IR magnets to provide reliable machine operation.

This paper analyzes and compares the thermal performance and the operation margin for IR quadrupoles based on $\mathrm{NbTi}$ and $\mathrm{Nb}_{3} \mathrm{Sn}$ superconductors with respect to radiation heat deposition. Results of experimental verification of the thermal calculation for $\mathrm{NbTi}$ IR quadrupoles, and possible measurements of operation margin of $\mathrm{Nb}_{3} \mathrm{Sn}$ IR quadrupoles are discussed.

Manuscript received August 28, 2006.

This work was supported by the U.S. Department of Energy.

Authors are with the Fermi National Accelerator Laboratory, Batavia, IL 60510 USA (I. Novitski phone: 630-840-4823; fax: 630-840-3369; e-mail: novitski@fnal.gov).

\section{THERMAL ANALYSIS}

\section{A. Magnet Design and ANSYS Thermal Model}

For a consistent comparison of $\mathrm{NbTi}$ and $\mathrm{Nb}_{3} \mathrm{Sn}$ IR quadrupoles the thermal analysis was performed for magnets with equivalent design and performance parameters. These IR quadrupoles were developed as candidates for the LHC IRs. Quadrupole cross-sections are shown in Fig. 1. Both magnets use two-layer coils and were designed for a maximum field gradient of $\sim 250 \mathrm{~T} / \mathrm{m}$. The details of magnet designs are reported in [6, 7].
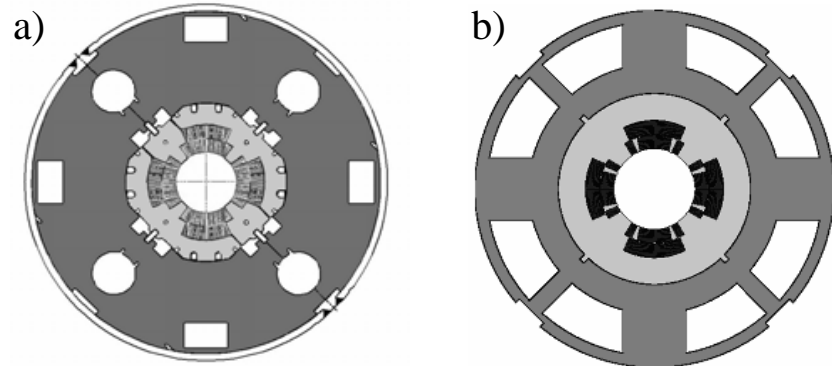

Fig. 1. 70 mm NbTi MQXB (a) and $90 \mathrm{~mm} \mathrm{Nb}_{3} \mathrm{Sn}$ (b) quad cross-sections.

The NbTi quadrupole coil has a 70-mm bore and is made of $15-\mathrm{mm}$ wide graded cable insulated with Kapton tape. The $\mathrm{Nb}_{3} \mathrm{Sn}$ quadrupole coil has a $90-\mathrm{mm}$ bore and uses $15-\mathrm{mm}$ wide cable insulated with thick S2-glass/epoxy insulation. In both designs the coil is supported by a stainless steel collar and surrounded by an iron yoke. The magnet cold mass, which includes coil, collar and yoke, is cooled with HeII at T=1.9 K.
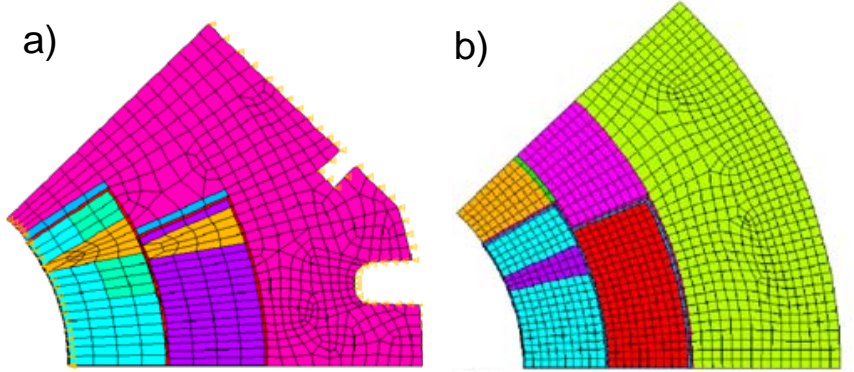

Fig. 2. 2D finite element thermal models: a) NbTi IRQ; b) $\mathrm{Nb}_{3} \mathrm{Sn}$ IRQ.

2D finite element thermal models of the collared coils were developed using ANSYS for both quadrupole designs. The models, shown in Fig. 2, are based on the octant symmetry and include inner and outer coils, interlayer and ground insulation, aluminum-bronze pole spacer and stainless steel collars [8]. 


\section{B. Material Properties \& Coil Cooling Conditions}

The thermal conductivity of materials at $1.9 \mathrm{~K}$ used in calculations is reported in Table I. For most of the magnet components the properties are well established. The thermal conductivity of the coils was calculated based on the cable structure and properties of its components. The azimuthal thermal conductivity includes the cable insulation. In the radial direction the cable insulation was added as a layer to the coil inner surface, the interlayer and outer-layer insulation. The calculated coil thermal conductivity values were compared with the available experimental data and were found consistent for similar samples [9]. Due to the small range of temperature variations the material properties were considered temperature independent in this analysis. This assumption leads to conservative results since the thermal conductivity of all materials used increases with the increasing temperature.

TABLE I. MATERIAL THERMAL CONDUCTIVITY @1.9K

\begin{tabular}{lll}
\hline \hline Material (component) & Thermal Conductivity at $1.9 \mathrm{~K}(\mathrm{~W} / \mathrm{m} / \mathrm{K})$ \\
\hline IRQ design & $70 \mathrm{~mm} \mathrm{NbTi}$ & $90 \mathrm{~mm} \mathrm{Nb}$ Sn \\
Inner Coil Azimuthal & 0.018 & 0.046 \\
Outer Coil Azimuthal & 0.016 & 0.046 \\
Inner Coil Radial & 4.54 & 10.0 \\
Outer Coil Radial & 6.45 & 10.0 \\
Copper (wedges) & 140 & - \\
Al Bronze (wedges, poles) & - & 0.8 \\
Kapton (insulation) & 0.005 (cable, ground) & 0.005 (ground) \\
S2-glass/epoxy (insulation) & - & 0.03 (cable) \\
Stainless Steel (collar) & 0.1 & 0.1 \\
\hline \hline
\end{tabular}

Boundary conditions include constant HeII temperature of $1.9 \mathrm{~K}$, and zero heat flux through the pole (45 degree line) and coil mid-plane. At the coil bore side, a constant heat transfer coefficient of $300 \mathrm{~W} / \mathrm{m}^{2} / \mathrm{K}$ was used (Kapitza resistance).

For the NbTi IR quadrupole, the case of inter-layer cooling channels was modeled by applying a temperature boundary condition of $1.95 \mathrm{~K}$ between the coil layers. This was not considered for the $\mathrm{Nb}_{3} \mathrm{Sn}$ IR quadrupole due to technological difficulties of channel implementation for a two-layer coil.

\section{Radiation Heat Deposition}

The energy deposition from secondary particles in the LHC IRs has been studied using MARS code [10]. For this analysis the 2D radial and azimuthal distribution of radiation heat deposition in the coil was approximated by the function that fits the MARS data for 70-mm NbTi quadrupole:

$$
P(r, \theta)=P o \cdot \exp \frac{-(r-\operatorname{Rin})}{R o} \cdot \frac{\theta o}{\theta+\theta o},
$$

where $r$ and $\theta$ are polar coordinates, Rin is the coil inner radius, $P o$ is the power deposited at the coil inner surface, $R o$ and $\theta o$ are fitting parameters.

Formula (1) was also used for the $90 \mathrm{~mm} \mathrm{Nb}_{3} \mathrm{Sn}$ quadrupole. It is clear that the real distribution of radiation heat deposition in IR quadrupoles may differ from (1) since it depends on the details of IR and magnet designs, material properties, etc. However, as it will be shown in the next sections, the details of the energy deposition profile are not too important for calculations of the magnet quench limit and operation margin since these parameters are determined by the average value of the heat deposition in coil turns.

\section{Temperature Profile}

The calculated 2D temperature profile for $\mathrm{NbTi}$ and $\mathrm{Nb}_{3} \mathrm{Sn}$ IR quadrupoles is shown in Figs. 3 and 4.

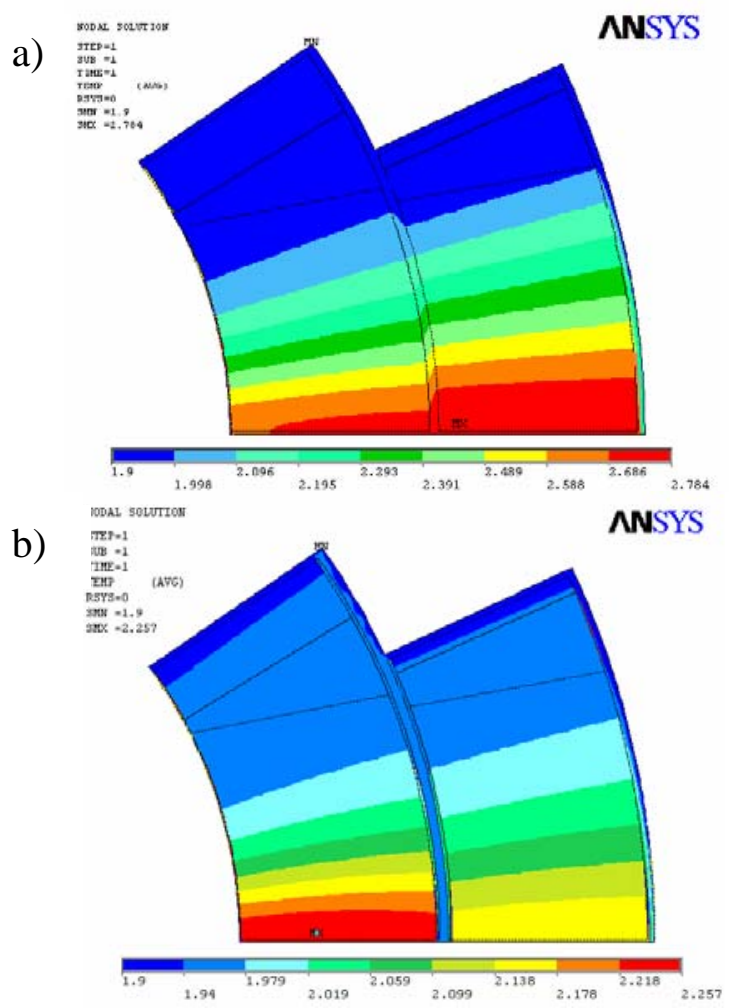

Fig.3. Calculated temperature profile in NbTi IR quadrupole for two cases: a) without and b) with the inter-layer HeII channel. In both cases it is assumed that HeII penetrates inside the collar blocks reaching the coil outer surface. 


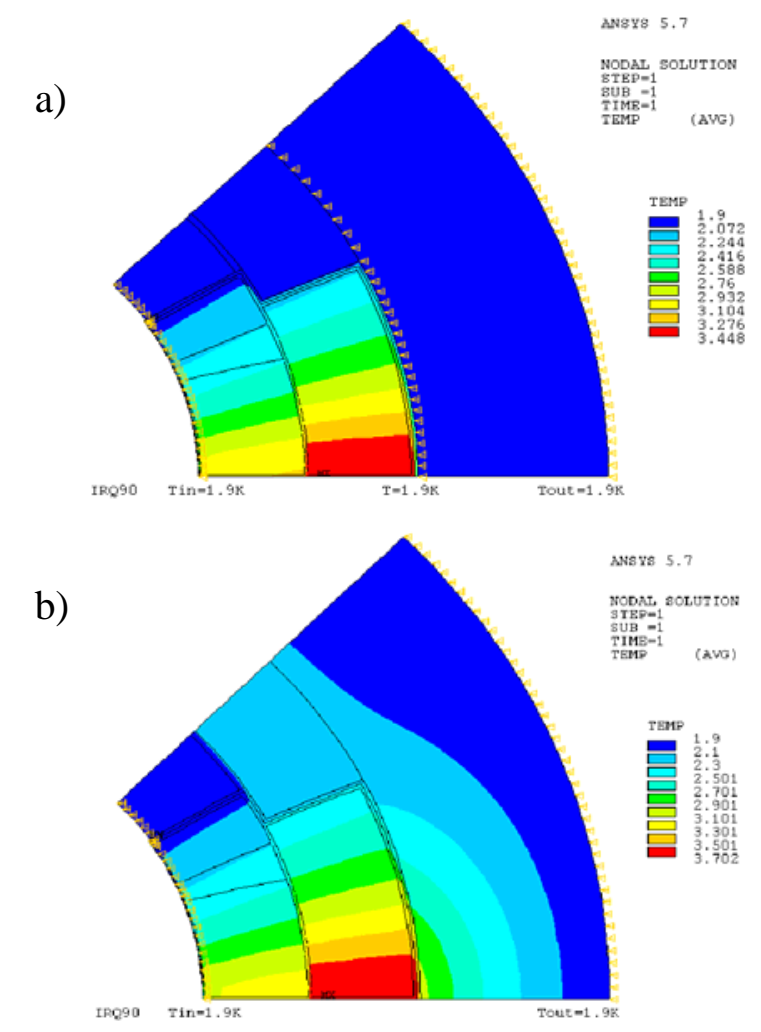

Fig.4. Calculated temperature profile in $\mathrm{Nb}_{3} \mathrm{Sn}$ IR quadrupole without the inter-layer channel for two cases: a) HeII penetrates between collars reaching the coil outer surface; and b) HeII does not penetrate inside collar blocks.

Based on formula (1) the radial and the azimuthal distributions of radiation heat deposition in the coil are substantially non-uniform. However, Figs. 3 and 4 show that only the azimuthal temperature profile in the coil is nonuniform while the radial temperature profile in each layer is quite uniform in both magnets due to large radial and small azimuthal thermal conductivity. In both cases the maximum turn temperature is in the coil mid-plane. It is determined by the average heat deposition power in the mid-plane turns.

The calculated temperature profiles along the coil mid-plane in $\mathrm{NbTi}$ and $\mathrm{Nb}_{3} \mathrm{Sn}$ IR quadrupoles for different coil cooling conditions are shown in Figs. 5 and 6. A larger temperature rise (especially in the coil outer layer) is observed when superfluid does not penetrate inside the collars. However, the effect is relatively small and the general temperature profile remains the same with the maximum in the outer layer. The interlayer cooling channel in the NbTi coil noticeably reduces the temperature of both layers. The temperature maximum in this case moves to the coil inner layer.

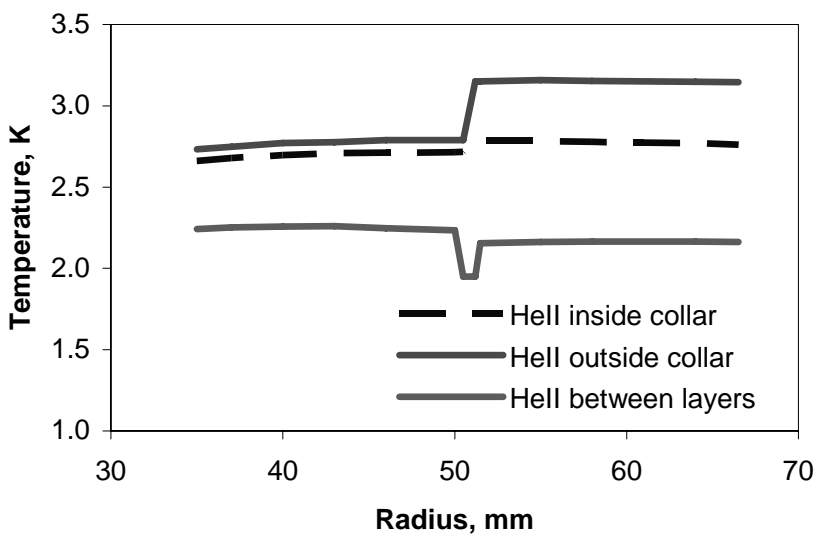

Fig.5. Temperature profile along the coil mid-plane in NbTi quadrupole.

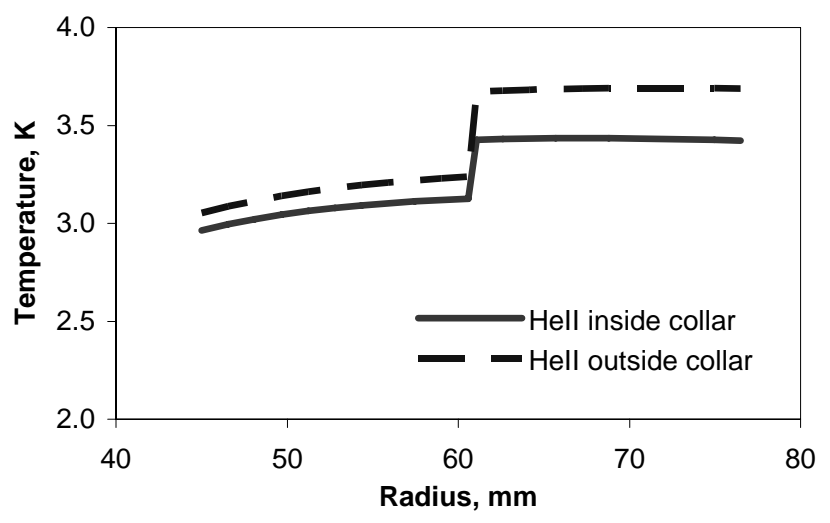

Fig.6. Temperature profile along the coil mid-plane in $\mathrm{Nb}_{3} \mathrm{Sn}$ quadrupole.

\section{QUENCH LIMIT AND OPERATION MARGIN}

The Magnet Operation Margin (MOM) with respect to the radiation heat deposition in the coil could be found as the minimum value of Turn Operation Margins (TOM) defined as

$$
\mathrm{TOM}_{i}=\mathrm{Pav}_{c_{-} \_} / \mathrm{Pav}_{t_{-} i} \text {, }
$$

where $\operatorname{Pav}_{c_{-} i}$ is the turn quench limit and $\operatorname{Pav}_{t_{-} i}$ is the average radiation heating power deposited in turn $i$.

The turn quench limit can be calculated as

$$
\operatorname{Pav}_{c_{-} i}=d T_{c_{-} i} / h\left(P_{a v_{-} i}\right),
$$

where $d T_{c_{-} i}$ is the turn critical temperature margin and $h\left(P_{a v_{-} i}\right)$ represents the turn cooling conditions in the coil.

Coefficient $h\left(P_{a v}\right)$ for each turn is determined from the temperature profile calculated in Section II for the distribution of heat deposition power in the coil given by (1). In the case of the temperature-independent material properties these coefficients are constants, determined only by the magnet design, turn insulation and the turn position in the coil. Coefficients $h\left(P_{a v}\right)$ for the inner-layer mid-plane turns of $\mathrm{NbTi}$ and $\mathrm{Nb}_{3} \mathrm{Sn}$ quadrupoles discussed here are 0.207 and $0.165 \mathrm{~K} \cdot \mathrm{cm}^{3} / \mathrm{mW}$ respectively. The $h\left(P_{a v}\right)$ values for both magnets are quite close. The thinner turn insulation in NbTi magnet with lower thermal conductivity produced the same effect as thicker but more conductive insulation in $\mathrm{Nb}_{3} \mathrm{Sn}$ magnet. 
The turn temperature margin $d T_{c \_i}$ is calculated based on the superconductor critical surface $I_{c}(B, T)$ and the turn current, operation temperature and magnetic field. Using the critical surface parameterizations for $\mathrm{NbTi}$ [11] and $\mathrm{Nb}_{3} \mathrm{Sn}$ [12] superconductors and the distribution of radiation heat deposition (1), the turn temperature margin, quench limit and operation margin were calculated for both quadrupoles. The magnet operating margin with respect to the radiation heat deposition, localized in the coil mid-planes, is determined by the operation margin of the inner-layer mid-plane turns.

The calculated quench limit at $\mathrm{T}=1.9 \mathrm{~K}$ for the inner-layer mid-plane turns of the $\mathrm{NbTi}$ and $\mathrm{Nb}_{3} \mathrm{Sn}$ IR quadrupoles vs. the magnet normalized operation current is presented in Fig. 7. One can see that the quench limit (and the corresponding magnet operation margin) strongly depends on superconductor used in the magnet, and can be also slightly varied by changing the magnet operation current with respect to its critical current. At $1.9 \mathrm{~K}$ the $\mathrm{Nb}_{3} \mathrm{Sn}$ quadrupole provides a quench limit a factor of 3.6 larger than for the $\mathrm{NbTi}$ quadrupole.

The Magnet Operation Margin vs. the average radiation power in the inner-layer mid-plane turn for the $\mathrm{NbTi}$ and $\mathrm{Nb}_{3} \mathrm{Sn}$ IR quadrupoles at $1.9 \mathrm{~K}$ and operation current $20 \%$ below the magnet critical current is plotted in Fig. 8. Based on the typical requirement for IR magnets to provide the operation margin of 3 , the average radiation power in the inner-layer mid-plane turns has to be limited by 3.3 and 13.3 $\mathrm{mW} / \mathrm{cm}^{3}$ in $\mathrm{NbTi}$ and $\mathrm{Nb}_{3} \mathrm{Sn}$ quadrupoles respectively.

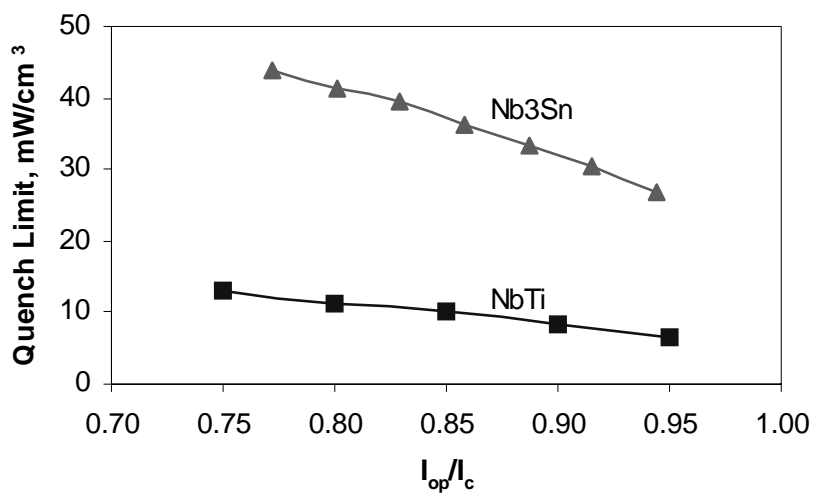

Fig. 7. Calculated quench limit at $\mathrm{T}_{\mathrm{Op}}=1.9 \mathrm{~K}$ for $\mathrm{Nb}_{3} \mathrm{Sn}$ and $\mathrm{NbTi}$ IRQ vs. the magnet operation current normalized on the magnet critical current.

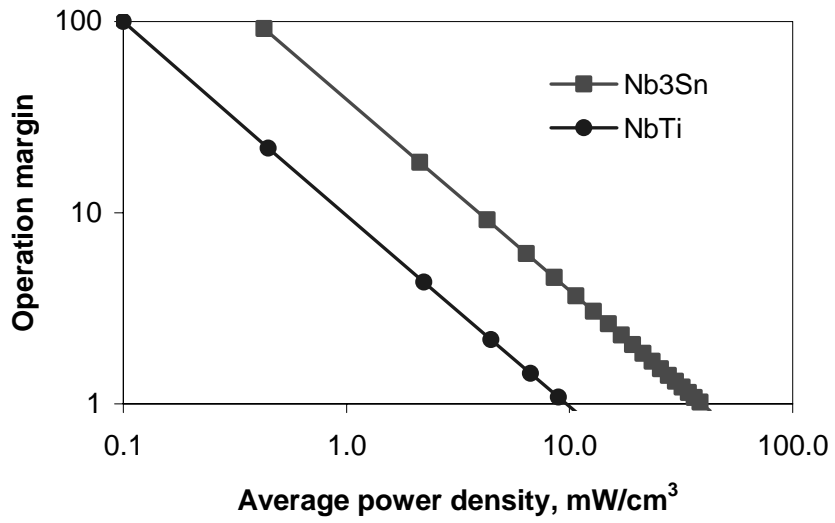

Fig. 8. Operation margin vs. the average power of energy deposition in the inner-layer mid-plane turn for the $\mathrm{NbTi}$ and $\mathrm{Nb}_{3} \mathrm{Sn}$ IR quadrupoles.

\section{Quench Limit Measurements}

Due to the large uncertainty in thermal conductivity of $\mathrm{NbTi}$ and $\mathrm{Nb}_{3} \mathrm{Sn}$ coils, results of the thermal analysis need an experimental verification. For the NbTi IR quadrupole the results described above were compared with the experimental data from thermal studies performed at Fermilab [13]. The studies were done using a special 2-m long NbTi quadrupole model designed to provide the level of AC losses in the coil comparable with the expected level of radiation heat deposition. Similar to radiation heat deposition, the AC loss power was mainly deposited in the coil inner layer with the maximum at high current ramp rates in the mid-plane turns.

The magnet quench limit was determined from measurements of the sensitivity of its critical current to the AC loss power in the coil. The quench current was measured as a function of the current ramp rate at currents $75-95 \%$ below the magnet maximum quench current. At the current ramp rates above $75 \mathrm{~A} / \mathrm{s}$ the quenches occurred in the inner-layer midplane turns. The measured AC loss power and its calculated distribution in the coil provided a correlation between the ramp rate and the power in the mid-plane turns.

The measured and calculated quench limit for the innerlayer mid-plane turns of $\mathrm{NbTi}$ quarupole at $1.9 \mathrm{~K}$ vs. the normalized magnet current is presented in Fig. 9. There is a good correlation of measured and calculated data in a wide range of magnet operation currents.

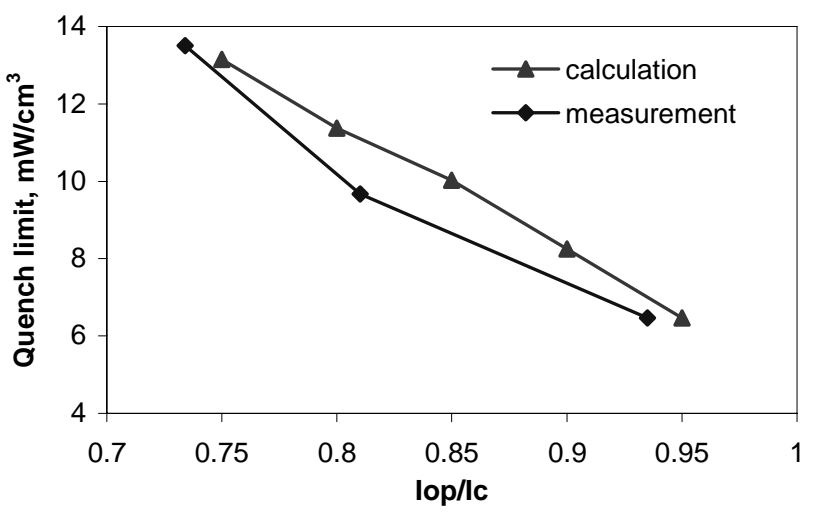

Fig. 9. Measured and calculated values of quench limit of inner-layer midplane turns for NbTi quarupole model at $1.9 \mathrm{~K}$ [13].

Experimental verification of the thermal analysis for $\mathrm{Nb}_{3} \mathrm{Sn}$ IR quadrupoles is also important. Due to difficulties with providing the controllable AC loss level and distribution in $\mathrm{Nb}_{3} \mathrm{Sn}$ coils these measurements will be performed using resistive heaters installed on the mid-plane turns before the coil impregnation with epoxy. The first successful measurement of the coil critical temperature at $\mathrm{I} / \mathrm{I}_{\mathrm{C}}>0.25$ using such heaters in $\mathrm{Nb}_{3} \mathrm{Sn}$ dipole models has been done [14].

\section{CONCLUSIONS}

Thermal analysis using 2D ANSYS models was performed for $\mathrm{NbTi}$ and $\mathrm{Nb}_{3} \mathrm{Sn}$ IR quadrupoles. It was shown that the $\mathrm{Nb}_{3} \mathrm{Sn}$ quadrupole at $1.9 \mathrm{~K}$ provides a factor of 3.6 larger operation margin with respect to radiation heat depositions than the NbTi quadrupole. The results of calculation for the $\mathrm{NbTi}$ quadrupole were compared with the available experimental data for similar model and demonstrated a good 
correlation. Experimental verification of the thermal analysis for $\mathrm{Nb}_{3} \mathrm{Sn}$ quadrupoles is essential and will be performed using special midplane heaters as soon as the appropriate model will be available.

\section{REFERENCES}

[1] A.D. McInturff et al., "The Fermilab Collider D0 Low- $\beta$ System", EPAC’1988, Rome, Italy, Vol.2, June 1988, p.1264.

[2] T. Shintomi et al., "Progress of LHC Low- $\beta$ Quadrupole Magnets at KEK”, IEEE Trans. on Applied Superconductivity, Vol.11, Issue 1, March 2001, p. 1562.

[3] N. Andreev et al., "Status of the LHC Inner Triplet Quadrupole Program at Fermilab”, IEEE Trans. on Applied Superconductivity, Vol.11, Issue 1, March 2001, p. 1558.

[4] S.A. Gourlay et al., "Magnet R\&D for the US LHC Accelerator Research Program (LARP)”, IEEE Trans. on Applied Superconductivity, Vol. 16, Issue 2, June 2006, p.324.

[5] R. Appleby et al., "The 2 mrad Crossing Angle Interaction Region and Extraction Line”, EPAC'2006, Edinburgh, Scotland, June 2006.

[6] R. Bossert et al., "Design, Development and Test of $2 \mathrm{~m}$ Model Magnets for the LHC Inner Triplet”, IEEE Trans. on Applied Superconductivity, Vol. 9, Issue 2, June 1999.

[7] A.V. Zlobin et al., "Conceptual Design Study of $\mathrm{Nb}_{3} \mathrm{Sn}$ Low-beta Quadrupoles for $2^{\text {nd }}$ Generation LHC IRs”, IEEE Transactions on Applied Superconductivity, Vol. 13, Issue 2, June 2003, p.1266.

[8] S. Yadav, A.V. Zlobin, "Finite Element Analysis of the Effect of Cooling Conditions on Two-Layer and Four-Layer HGQ Magnet Designs”, Technical note TD-02-047, Fermilab, December 13, 2002.

[9] L. Imbasciati et al., "Thermo-Mechanical Characterization of Insulated and Epoxy-Impregnated $\mathrm{Nb}_{3} \mathrm{Sn}$ Composites", IEEE Trans. on Applied Superconductivity, Vol. 13, Issue 2, June 2003, p.1788.

[10] N.V. Mokhov and J.B. Strait, "Towards the Optimal LHC Interaction Region: Beam-Induced Energy Deposition”, Proc. Of Particle Accelerator Conference (PAC’97), Vancouver, Canada, May 12-17, 1997, Vol.1, p.124.

[11] M.A. Green, "Calculating the Jc, B, T surface for NbTi using a reducedstate model”, IEEE Trans. on Magnetics, Vol. 25, Issue 2, 1989, p.2119.

[12] L.T. Summers et al., "A model for the prediction of $\mathrm{Nb}_{3} \mathrm{Sn}$ critical current as a function of field, temperature, strain, and radiation damage," IEEE Trans. on Magnetics, Vol. 27, Issue 2, March 1991, p.2041.

[13] L. Chiesa et al. "Thermal Studies of a High Gradient Quadrupole Magnet Cooled with Pressurized, Stagnant Superfluid,” IEEE Trans. on Applied Superconductivity, Vol. 11, Issue 1, March 2001, p.1625.

[14] S. Feher et al., "Test Results of Shell-Type $\mathrm{Nb}_{3} \mathrm{Sn}$ Dipole Coils", IEEE Trans. on Applied Superconductivity, Vol. 14, Issue 2, June 2004, p.349. 